\title{
The Co-Evolution of Supermassive Black Holes and Galaxies: Observational Constraints
}

\author{
Xian Zhong Zheng \\ Purple Mountain Observatory, Chinese Academy of Sciences, \\ 2 West-Beijing Road, Nanjing 210008, P. R. China \\ email: xzzheng@pmo.ac.cn
}

\begin{abstract}
The connection between the growth of supermassive black holes (SMBHs) and the assembly of their host galaxies is termed 'co-evolution'. Understanding co-evolution is one of the most fundamental issues in modern astrophysics. In this contribution, we review recent progress in addressing how the growth of SMBHs is linked to the properties of their host galaxies in the context of galaxy evolution, from the observational point of view. Although a coherent picture has not yet emerged, multiple pathways of co-evolution appear to be favored with a probable dependence on AGN luminosity and redshift.
\end{abstract}

Keywords. galaxies: general, galaxies: evolution, galaxies: nuclei, quasars: general

\section{Introduction}

In the past two decades great effort had been made to understand the origin of the tight correlation between the mass of supermassive black holes (SMBHs) and velocity dispersion/bulge stellar mass in nearby massive galaxies. The SMBHs are often believed to couple only with bulges and have no relationship with the mass of the underlying dark matter halo (Kormendy \& Bender 2011; Beifiori et al. 2012) or with pseudobulges/disks (Kormendy et al. 2011). The energetic output of growing SMBHs, seen as AGN, can affect their surrounding galaxies/environments (Fabian 2012 and references therein). AGN feedback from SMBHs is taken as a more effective mechanism of quenching gas cooling and star formation in the framework of galaxy formation and evolution (Somervile et al. 2008; Silk \& Mamon 2012), although its physical processes are poorly understood. Recently, observational evidence accumulates towards a non-universal $M-\sigma_{*}$ relation (e.g., Gultekin et al. 2009; Greene et al. 2010; McConnell et al. 2011), implying complications for the interaction between SMBHs and galaxies. These findings and constraints from observations provide new insights in exploring multiple aspects of the co-evolution and its processes (Schawinski 2012).

\section{Statistical Links between AGN and Star Formation Activity}

Deep multi-wavelength surveys over a large area provide a complete census of galaxy and AGN populations out to $z \sim 4$. This is particularly true for $z<1$. It has been pointed out by a number of studies that the global BH accretion history traces the global galaxy star formation history. The latter is indeed the history of galaxy stellar mass growth. An up-to-date comparison between the two is presented in Zheng et al. (2009) (Fig. 1). In particular, the two activities also trace each other in intensity, following a mode of so-called 'downsizing'. The match between $\mathrm{BH}$ accretion rate and star formation rate 


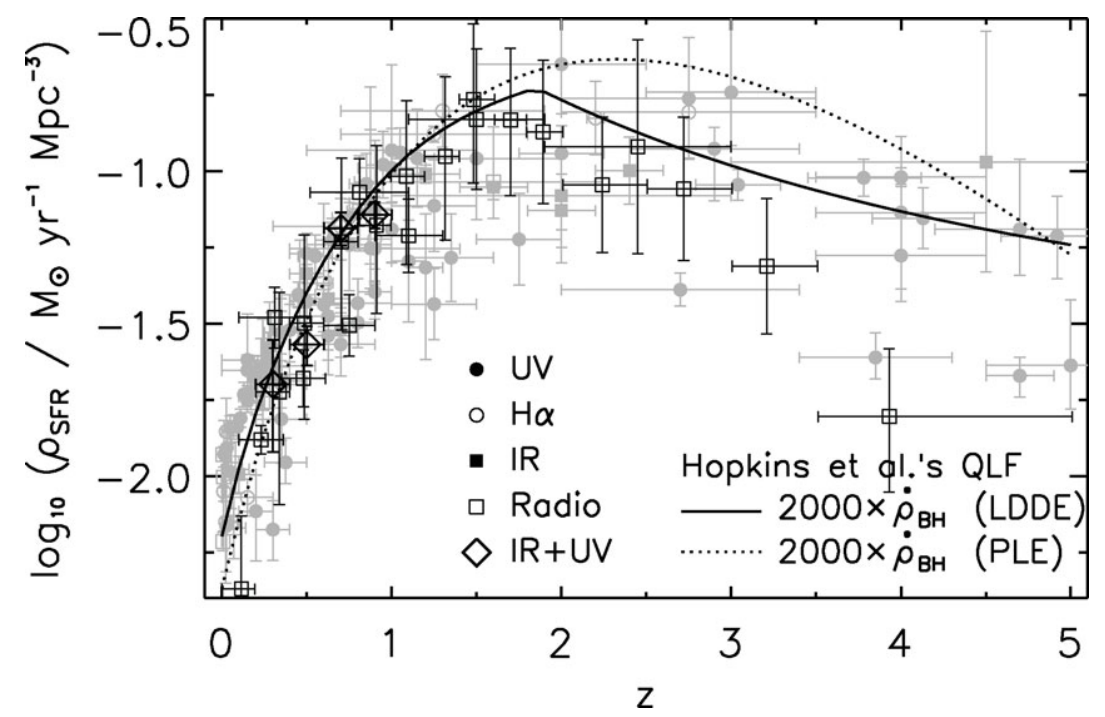

Figure 1. The global similarity of cosmic star formation history and cosmic BH accretion history. The proportion of 2000 between the two is consistent with the local SMBH-bulge mass relation accounting for the gas recycling of a factor 2 due to stellar evolution (Zheng et al. 2009).

suggests that the duty cycle of AGN is about two orders of magnitude shorter than that of starbursts. The integral of the growth of SMBHs and the growth of stellar mass through star formation results in a BH-bulge mass ratio consistent with the local $M-\sigma_{*}$ relation.

The global and differential similarities of AGN and starburst activity are only valid in a statistical sense. They are not necessarily linked on a one-to-one basis. The star-forming galaxies hosting the vast majority of cosmic star formation are indeed dominated by disk galaxies out to $z \sim 2$ (Wuyts et al. 2011). The enhanced star formation induced by mergers represents only a small fraction of the overall SFR at $z \sim 2$ (Rodighiero et al. 2011) and $z<1$ (Guo et al. 2011). This is also confirmed by direct measurement of SFRs in close pairs and mergers (Robaina et al. 2009; Jogee et al. 2009). It becomes clear that starburst events mostly take place in isolated disks at least out to $z \sim 2$. These facts rule out the simplest co-evolution picture that $\mathrm{BH}$ accretion and bulge growth through star formation take place simultaneously in the same event. If a delay exists between $\mathrm{BH}$ accretion and starburst in a galaxy (Hopkins 2012), one would not see a direct link between the two processes from observations but SMBHs and bulges could grow in a proportional manner. Such a picture is also ruled out because starbursts are mostly associated with isolated disks other than growing bulges. The similarity between the global accretion history of SMBHs and the star formation history of galaxies is likely controlled by gas supply, which declines rapidly with cosmic time since $z \sim 2$. However, processes triggering both AGN activity and star formation remain to be understood (Kauffmann et al. 2007).

The local $M-\sigma_{*}$ relation involves mass and morphology (i.e., bulges/spheroids). A bulge can be built up through star formation and/or merging stars from, e.g., its surrounding disk. The bulk of stars formed in isolated disks needs to be transformed into bulges via certain physical processes (e.g., mergers, disk instability or secular processes) in order to meet the local $M-\sigma_{*}$ relation. The buildup of bulges has been associated with the quenching of star formation (Cheung et al. 2012). What are the processes governing the growth of bulges in different galaxy populations at different cosmic epochs? 


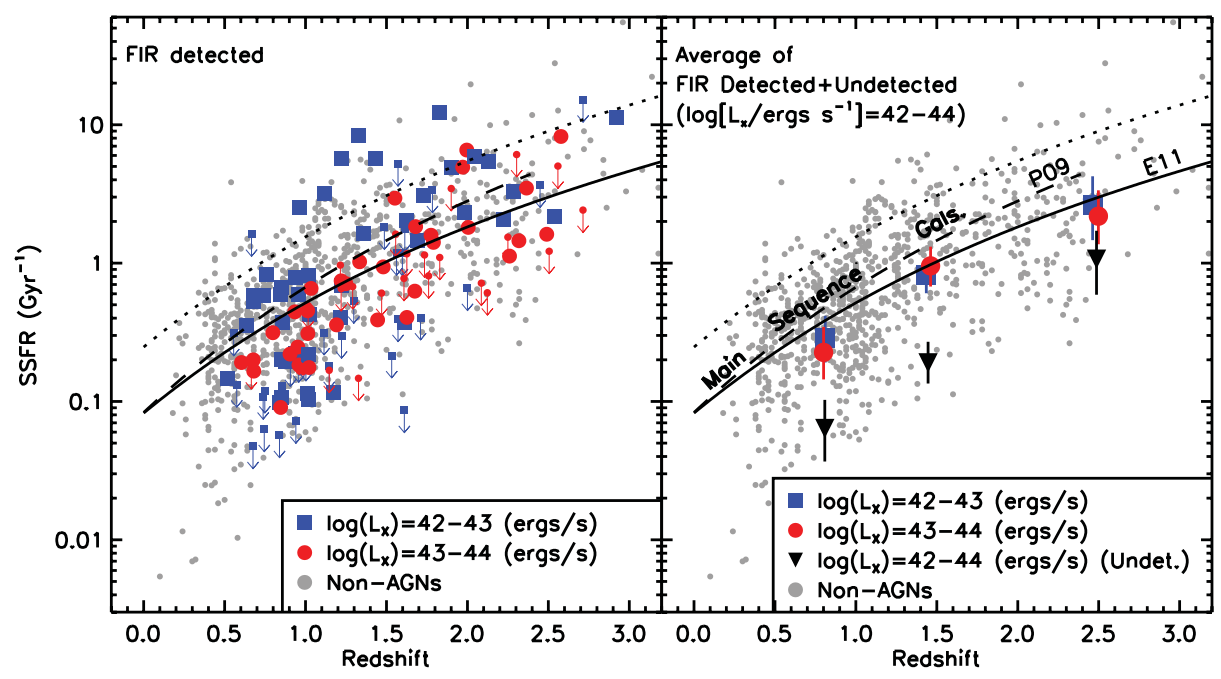

Figure 2. Comparison of the specific SFR between the host galaxies of moderate-luminosity $\mathrm{X}$-ray selected AGN and normal star-forming galaxies over $0<z<3$ (Mullaney et al. 2012). The increase in sSFR of the AGN host galaxies with redshift follows the increase in the global sSFR of all galaxies. No correlation is seen between AGN luminosity and sSFR.

Are these processes universally responsible for the ignition of AGN activity and the establishment of the $M-\sigma_{*}$ relation? What are the key mechanisms over galactic scales for transporting materials to the center of a galaxy and feeding of the central black hole? The relationship between AGN activity and the properties of their host galaxies hold the key to answering these questions.

\section{The Properties of AGN Host Galaxies}

The presence of AGN affects the determination of the properties of the host galaxy, especially for the most luminous ones. Relevant studies often target moderate-luminosity AGN in order to minimize contamination from the AGN. On the other hand, the diversity of the AGN population induces uncertainties in the selection of AGN samples hindering the derivation of a consistent picture

Star formation and stellar mass. The AGN host galaxies are found to exhibit a star formation activity similar to the entire galaxy population in a statistical manner (Fig. 2; Mullaney et al. 2012; see also Shao et al. 2010). Silverman et al. (2009) noted that the star formation rate of the host galaxies does not correlate with AGN luminosity (Fig. 3). This is mostly true for the low- and moderate-luminosity AGN. In contrast, the most luminous AGN have star formation rates likely enhanced by merger events (Rosario et al. 2012). The incidence of AGN strongly depends on galaxy stellar mass in the sense that AGN are preferentially found in massive galaxies, but the BH accretion mode seems independent of both the stellar mass and color of the host galaxy (Aird et al. 2012; Bongiorno et al. 2012). All together, one may conclude that the occurrence of an AGN involves a massive galaxy and sufficient gas supply to fuel both the AGN and star formation; the presence of an AGN seems neither to be related to quenching of star formation nor to have significant influence on the properties of the host galaxy.

Morphologies. Large studies of the morphologies of AGN host galaxies are attributed to the low-/moderate-luminosity AGN. These AGN are mostly found in disk-dominated 


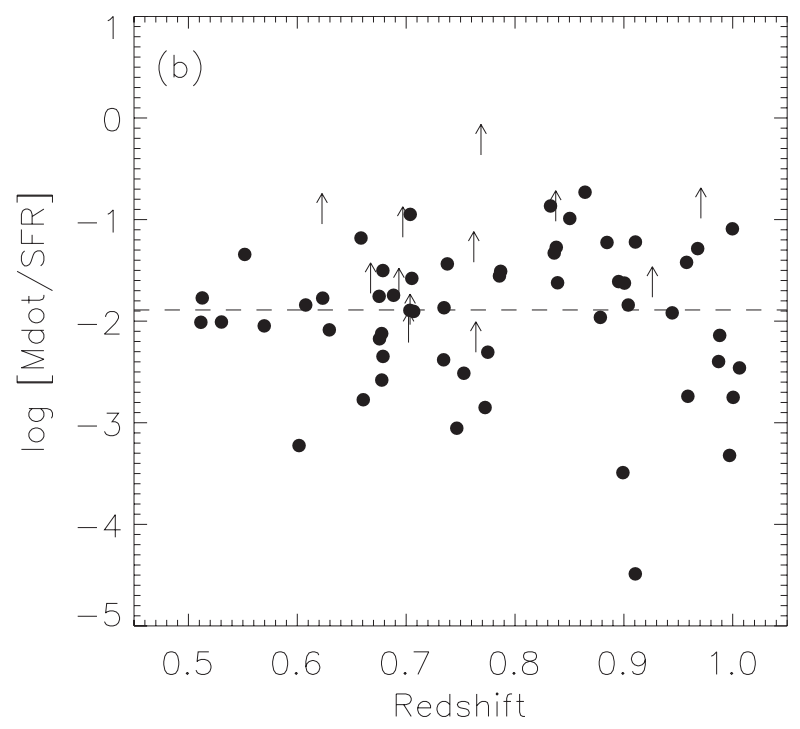

Figure 3. Comparison between $\mathrm{BH}$ accretion rate and star formation rate of the host galaxies of X-ray-selected AGN from zCOSMOS (Silverman et al. 2009). It is clearly seen that there is no correlation or anti-correlation of intensity between AGN and star formation activity over the redshift range examined.

galaxies other than mergers/interacting galaxies out to $z \sim 2$. The AGN host galaxies tend to be systematically more concentrated than normal galaxies at $z<1$ (Grogin et al. 2005; Geogakakis et al. 2008; Cisternas et al. 2011), and indistinguishable from the galaxy population at $z \sim 2$ (Fig. 4; Schawinski et al. 2011). The latter is confirmed by Kocevski et al. (2012) using HST/WFC3 imaging from the CANDELS survey. It is now convincingly established that stochastic accretion of gas, instead of violent dynamical processes (major mergers/interactions), plays the major role regarding the fuelling of low-/moderate-luminosity AGN over cosmic time at least out to $z \sim 2$. The stochastic accretion is argued to be driven by secular processes in the host galaxies. The morphologies of galaxies hosting the most luminous AGN, known as quasars, are less explored because of glare from the central AGN over the host galaxy (e.g., Bahcall et al. 1997). Major mergers are believed to play a crucial role in triggering the most luminous AGN (Treister et al. 2012), although the role of violent events is possibly less important at $z>1$ (Rosario et al. 2012).

The BH-galaxy mass ratios. One critical question to understand the co-evolution of SMBHs and galaxies is which one grew first. This is vital to ascertaining which of the two components regulates the other (Volonteri 2012). Only indirect measurements of the BH-galaxy mass ratio are feasible for AGN over cosmic distances. SMBHs in distant AGN are often found to be more massive at fixed galaxy mass than the predictions based on the local BH-bulge mass relation, suggesting SMBHs to be in place at earlier times during the galaxy formation process (Fig. 5, Bennert et al. 2011; Merloni et al. 2010). The scatter in the BH-galaxy mass ratio appears to be rather large for galaxies with growing SMBHs, illustrating the complexities of the pathways that govern the growth of SMBHs and bulges (Bennert et al. 2011, Greene et al. 2010). 


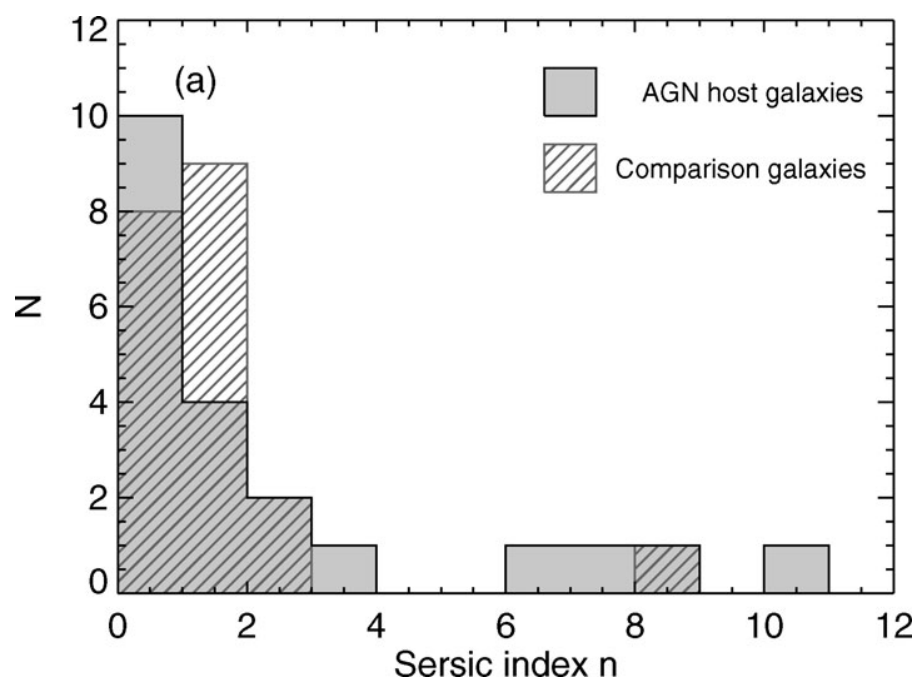

Figure 4. The distribution of Sersic index derived from rest-frame optical imaging for AGN hosts and comparison galaxies at $z \sim 2$, suggesting that the AGN host galaxies are dominated by late-type systems with low Sersic indices and indistinguishable from the parent galaxy population (Schawinski et al. 2011).

\section{Multiple Evolutionary Pathways}

The links between AGN and star formation activity, along with the connections between AGN activity and the properties of AGN host galaxies provide important observational constraints on the SMBH-galaxy co-evolution. In particular,

- the gas supply is key to the fuelling of AGN and star formation within galaxies;

- stochastic accretion of gas is commonly seen in disk-dominated galaxies over cosmic time. $\mathrm{BH}$ accretion does not require a bulge;

- secular processes are predominantly responsible for triggering and feeding moderateluminosity AGN;

- the growth of SMBHs preceding the assembly of galaxies hints that processes feeding AGN are easier to happen at $z \gtrsim 2$;

- disk disruption plays an important role in driving bulge growth and the evolution of the BH-bulge mass relation;

- major mergers are preferentially associated with the most luminous AGN.

The observational evidence points to multiply evolutionary pathways for massive galaxies satisfying the local $M-\sigma_{*}$ relation. The SMBH-galaxy co-evolution is neither universally a "hand-in-hand dance" nor a growth in lock step with a delay in occurrence time.

Merger-driven scenario. Most luminous AGN are exclusively hosted by major mergers in contrast to moderate- and low-luminosity AGN, which are mostly associated with normal galaxies (Treister et al. 2012), suggesting that the triggering and fuelling mechanism is likely a function of AGN luminosity. The mechanisms involving violent mergers may be more efficient in producing luminous AGN compared to stochastic fuelling of SMBHs in disk galaxies. This is supported by the finding of distinct $\mathrm{BH}$ accretion modes with respect to the dependence on luminosity among local AGN (Kauffmann et al. 2009) and AGN at intermediate redshifts (Rosario et al. 2012). Regarding merger-driven evolution in self-regulated models, the growth of the $\mathrm{SMBH}$ and the formation of the bulge are closely linked through feedback or feeding (Di Matteo et al. 2005; Hopkins et al. 2008; 


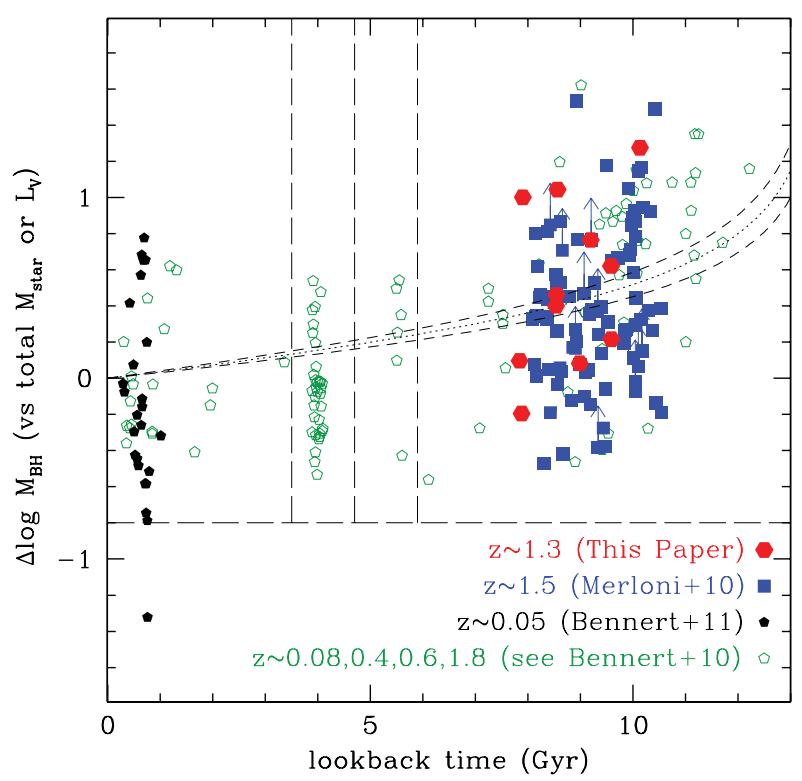

Figure 5. The evolution of BH-to-galaxy mass ratio of AGN with respect to the local relation of AGN (Bennert et al. 2011). The higher BH-to-galaxy mass ratio at increasing redshift suggests that $\mathrm{BH}$ growth precedes galaxy assembly.

Somerville et al. 2008). How AGN feedback affects the quenching of star formation in bulges remains an open question. This picture represents one possible pathway in the formation of massive galaxies.

Secular evolution. The majority of moderate-luminosity AGN is fuelled by internal mechanisms rather than violent mergers, suggesting that secular evolution plays a crucial role in regulating the growth of SMBHs in disk-dominated galaxies. The disk instabilities could be an important AGN feeding mechanism at $z>1$. The AGN activity at $z \sim$ 2 are mostly regulated by secular processes and supposed to be ideal for probing the conditions in these processes. In this scenario, the growing SMBHs reside in bulgeless galaxies (Simmons et al. 2012; Greence et al. 2010).

Non-causal evolution. The SMBH-galaxy correlation can, arguably, be caused as the natural product of hierarchical galaxy evolution, regardless of the merger history of the galaxy (Peng 2007; Jahnke \& Maccio 2011). This correlation can be obtained even without an apparent causation between the growth of SMBHs and galaxy assembly. Hence, in this scenario, SMBH growth and galaxy growth need not be coupled through direct feedback processes (Cen 2012).

\section{Implications from the assembly of massive galaxies}

It is likely that outliers on the $M-\sigma_{*}$ relation provide the key clues to understanding the connection between SMBHs and galaxies. It is found that Brightest Cluster Galaxies (BCGs) show systematic offsets from the correlation in Fig.6 (McConnell et al. 2011). This could point to unique evolutionary processes near the highest density peaks in the Universe. The growth of the most massive galaxies can be characterized by two stages: an early one that forms the central part at $z>2$, and a later phase that builds up the outer stellar halo via continuously accreting satellite galaxies (van Dokkum et al. 2010). The formation of the outer halo is unlikely to fuel $\mathrm{BH}$ accretion and to disturb significantly the kinematics of stars. Therefore, these BCGs with a higher BH-to-bulge mass ratio at 


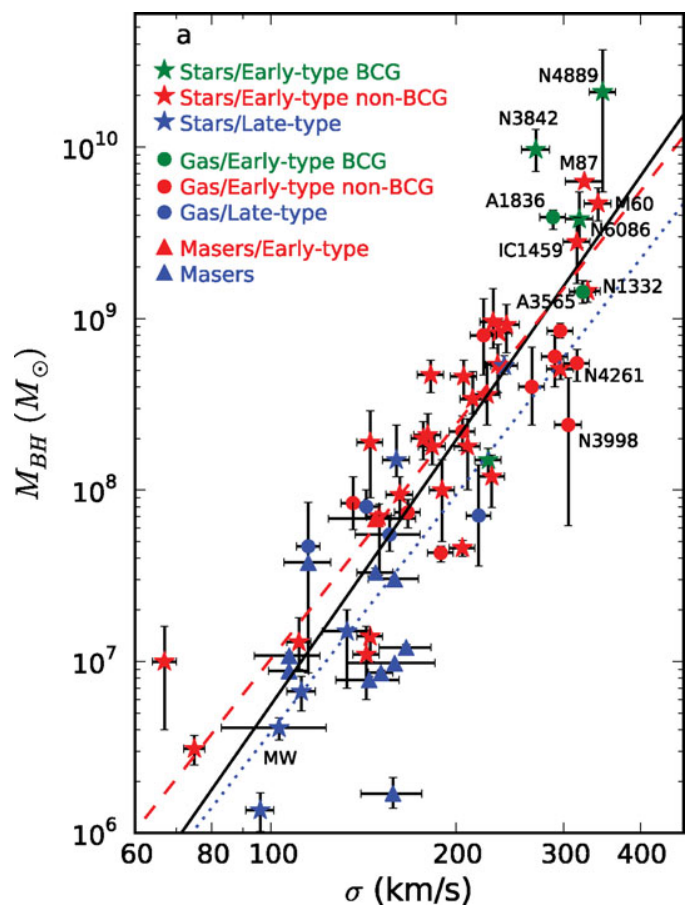

Figure 6. SMBH mass versus velocity dispersion of host galaxies from McConnell et al. (2011). The Brightest Cluster Galaxies (BCGs) appear to contain SMBHs much more massive than the predicted by the $M-\sigma_{*}$ relation.

$z \sim 2$ could evolve little in the $M-\sigma_{*}$ relation since then, appearing as the outliers with respect to the less massive galaxies.

\section{Acknowledgments}

I am very thankful to the IAU Symposium 295 organizers, in particular Daniel Thomas, Anna Pasquali and Ignacio Ferreras, for inviting me to give the targeted talk, and for organizing such a wonderful meeting. It has been a great experience for me.

\section{References}

Aird, J., Coil, A. L., Moustakas, J., et al. 2012, ApJ, 746, 90

Alexander, D. M. \& Hickox, R. C. 2012, New Astronomy, 56, 93

Bahcall, J. N., Kirhakos, S., Saxe, D. H., \& Schneider, D. P. 1997, ApJ, 479, 642

Beifiori, A., Courteau, S., Corsini, E. M., \& Zhu, Y. 2012, MNRAS, 419, 2497

Bennert, V. N., Auger, M. W., Treu, T., Woo, J.-H., \& Malkan, M. A. 2011, ApJ, 742, 107

Bogdán, Á., Forman, W. R., Zhuravleva, I., et al. 2012, ApJ, 753, 140

Bongiorno, A., Merloni, A., Brusa, M., et al. 2012, MNRAS, 427, 3103

Cen, R. 2012, ApJ, 755, 28

Cheung, E., Faber, S. M., Koo, D. C., et al. 2012, ApJ, 760, 131

Cisternas, M., Jahnke, K., Inskip, K. J., et al. 2011, ApJ, 726, 57

Di Matteo, T., Springel, V., \& Hernquist, L. 2005, Nature, 433, 604

Fabian, A. C. 2012, ARAA, 50, 455

Georgakakis, A., Coil, A. L., Laird, E. S., et al. 2009, MNRAS, 397, 623

Greene, J. E., Peng, C. Y., Kim, M., et al. 2010, ApJ, 721, 26 
Grogin, N. A., Conselice, C. J., Chatzichristou, E., et al. 2005, ApJ, 627, L97

Guo, K., Zheng, X. Z., \& Fu, H. 2011, Galaxy Evolution: Infrared to Millimeter Wavelength Perspective, 446, 145

Gültekin, K., Richstone, D. O., Gebhardt, K., et al. 2009, ApJ, 698, 198

Hopkins, P. F., Hernquist, L., Cox, T. J., \& Kereš, D. 2008, ApJS, 175, 356

Hopkins, P. F., Younger, J. D., Hayward, C. C., Narayanan, D., \& Hernquist, L. 2010, MNRAS, 402, 1693

Hopkins, P. F. 2012, MNRAS, 420, L8

Jahnke, K. \& Macciò, A. V. 2011, ApJ, 734, 92

Jogee, S., Miller, S. H., Penner, K., et al. 2009, ApJ, 697, 1971

Kauffmann, G. \& Heckman, T. M. 2009, MNRAS, 397, 135

Kauffmann, G., Heckman, T. M., Budavári, T., et al. 2007, ApJS, 173, 357

Kocevski, D. D., Faber, S. M., Mozena, M., et al. 2012, ApJ, 744, 148

Kormendy, J. \& Bender, R. 2011, Nature, 469, 377

Kormendy, J., Bender, R., \& Cornell, M. E. 2011, Nature, 469, 374

McConnell, N. J., Ma, C.-P., Gebhardt, K., et al. 2011, Nature, 480, 215

Merloni, A., Bongiorno, A., Bolzonella, M., et al. 2010, ApJ, 708, 137

Mullaney, J. R., Pannella, M., Daddi, E., et al. 2012, MNRAS, 419, 95

Peng, C. Y. 2007, ApJ, 671, 1098

Robaina, A. R., Bell, E. F., Skelton, R. E., et al. 2009, ApJ, 704, 324

Rodighiero, G., Daddi, E., Baronchelli, I., et al. 2011, ApJ, 739, L40

Rosario, D. J., Santini, P., Lutz, D., et al. 2012, A\& $A$, 545, A45

Schawinski, K., Treister, E., Urry, C. M., et al. 2011, ApJ, 727, L31

Schawinski, K., 2011 Frank N. Bash Symposium, "New Horizons in Astronomy", arXiv:1206.2661

Shao, L., Lutz, D., Nordon, R., et al. 2010, A\&A, 518, L26

Silk, J. \& Mamon, G. A. 2012, Research in Astronomy and Astrophysics, 12, 917

Silverman, J. D., Lamareille, F., Maier, C., et al. 2009, ApJ, 696, 396

Simmons, B. D., Lintott, C., Schawinski, K., et al. 2013, MNRAS, 429, 2199

Somerville, R. S., Hopkins, P. F., Cox, T. J., Robertson, B. E., \& Hernquist, L. 2008, MNRAS, 391,481

Treister, E., Schawinski, K., Urry, C. M., \& Simmons, B. D. 2012, ApJ, 758, L39

Treuthardt, P., Seigar, M. S., Sierra, A. D., et al. 2012, MNRAS, 423, 3118

van Dokkum, P. G., Whitaker, K. E., Brammer, G., et al. 2010, ApJ, 709, 1018

Volonteri, M. 2012, Science, 337, 544

Zheng, X. Z., Bell, E. F., Somerville, R. S., et al. 2009, ApJ, 707, 1566 